\title{
Calabi-Yau domains in three manifolds
}

\author{
Francisco Martín* William H. Meeks, III ${ }^{\dagger}$
}

September 11, 2021

\begin{abstract}
We prove that for every smooth compact Riemannian three-manifold $\bar{W}$ with nonempty boundary, there exists a smooth properly embedded one-manifold $\Delta \subset W=\operatorname{Int}(\bar{W})$, each of whose components is a simple closed curve and such that the domain $\mathcal{D}=W-\Delta$ does not admit any properly immersed open surfaces with at least one annular end, bounded mean curvature, compact boundary (possibly empty) and a complete induced Riemannian metric.
\end{abstract}

2000 Mathematics Subject Classification. Primary 53A10; Secondary 49Q05, 49Q10, 53C42. Key words and phrases: Complete bounded minimal surface, proper minimal immersion, CalabiYau problem for minimal surfaces.

\section{Introduction.}

A natural question in the global theory of minimal surfaces, first raised by Calabi in 1965 [2] and later revisited by Yau [11, 12], asks whether or not there exists a complete immersed minimal surface in a bounded domain $\mathcal{D}$ in $\mathbb{R}^{3}$. As is customary, we will refer to this problem as the Calabi-Yau problem for minimal surfaces. In 1996, Nadirashvili [10] provided the first example of a complete, bounded, immersed minimal surface in $\mathbb{R}^{3}$. However, Nadirashvili's techniques did not provide properness of such a complete minimal immersion in any bounded domain. Under certain restrictions on $\mathcal{D}$ and the topology of an open surface 1 1 $M$, Alarcón, Ferrer, Martín, and Morales [1, 7, 8, 9] proved the existence of a complete, proper minimal immersion of $M$ in $\mathcal{D}$. Recently, Ferrer, Martin and Meeks [4] have given a complete solution to the "proper Calabi-Yau problem for smooth bounded domains" by demonstrating that for every smooth bounded domain $\mathcal{D} \subset \mathbb{R}^{3}$ and for every open surface $M$, there exists a complete proper minimal immersion $f: M \rightarrow \mathcal{D}$; furthermore, in [4], they proved that such an immersion $f: M \rightarrow \mathcal{D}$ can be constructed so that for any two distinct ends $E_{1}, E_{2}$ of $M$, the limit sets $L\left(E_{1}\right), L\left(E_{2}\right)$ in $\partial \mathcal{D}$ are disjoint compact set 2 .

In contrast to the above existence results, in this paper we prove the existence of nonsmooth bounded domains $\mathcal{D}$ in $\mathbb{R}^{3}$, and more generally, domains $\mathcal{D}$ inside any Riemannian three-manifold, for which some open surface $M$ can not be properly immersed into $\mathcal{D}$ as a complete surface with bounded mean curvature. In this case, we will say that $\mathcal{D}$ is a Calabi-Yau domain for $M$. The result described

\footnotetext{
${ }^{*}$ This research is partially supported by MEC-FEDER Grant no. MTM2007 - 61775.

${ }^{\dagger}$ This material is based upon work for the NSF under Award No. DMS - 0703213. Any opinions, findings, and conclusions or recommendations expressed in this publication are those of the authors and do not necessarily reflect the views of the NSF.

${ }^{1}$ We say that a surface is open if it is connected, noncompact and without boundary.

${ }^{2}$ See Definition 2.1 for the definition of the limit set of an end of a surface in a three-manifold.
} 
in the next theorem generalizes the main theorem of Martín, Meeks and Nadirashvili in [6] which demonstrates the existence of nonsmooth bounded domains in $\mathbb{R}^{3}$ which do not admit any complete, properly immersed minimal surfaces with compact boundary (possibly empty) and at least one annular end.

Theorem 1.1 Let $\bar{W}$ be a smooth compact Riemannian three-manifold with nonempty boundary and let $W=\operatorname{Int}(\bar{W})$. There exists a properly embedded one-manifold $\Delta \subset W$ whose path components are smooth simple closed curves, such that $\mathcal{D}=W-\Delta$ is a Calabi-Yau domain for any surface with compact boundary (possibly empty) and at least one annular end. In particular, $\mathcal{D}$ does not admit any complete, noncompact, properly immersed surfaces of finite topology, compact boundary and constant mean curvature.

\section{Notation and the description of $\Delta$.}

Before proceeding with the proof of the main theorem, we fix some notation.

1. $\mathbb{B}(R)=\left\{x \in \mathbb{R}^{3}|| x \mid<R\right\}$ and $\mathbb{B}=\mathbb{B}(1)$.

2. $\overline{\mathbb{B}(R)}=\left\{x \in \mathbb{R}^{3}|| x \mid \leq R\right\}$ and $\overline{\mathbb{B}}=\overline{\mathbb{B}(1)}$.

3. $\mathbb{S}^{2}(R)=\partial \mathbb{B}(R)$ and $\mathbb{S}^{2}=\partial \mathbb{B}$.

4. For $p \in \mathbb{R}^{3}$ and $\varepsilon>0, \mathbb{B}(p, \varepsilon)=\left\{x \in \mathbb{R}^{3} \mid d(p, x)<\varepsilon\right\}$ is the open ball of radius $\varepsilon$ centered at $p$.

5. For $n \in \mathbb{N}, \mathbb{B}_{n}=\mathbb{B}\left(1-\frac{1}{2^{n}}\right)$ and $\mathbb{S}_{n}^{2}=\partial \mathbb{B}_{n}$.

6. For any set $F \subset \mathbb{R}^{3}$, the cone on $F$ is

$$
C(F)=\left\{x \in \mathbb{R}^{3} \mid x=t a \text { where } t \in(0, \infty) \text { and } a \in F\right\} .
$$

7. For any set $F \subset \mathbb{R}^{3}$ and $\varepsilon>0$, let $F(\varepsilon)=\left\{x \in \mathbb{R}^{3} \mid d(x, F) \leq \varepsilon\right\}$ be the closed $\varepsilon$ neighborhood of $F$, where $d$ is the distance function in $\mathbb{R}^{3}$.

In the proof of Theorem 1.1, we will need the following definition.

Definition 2.1 Let $f: M \rightarrow \mathcal{D}$ be a proper immersion of surface $M$ with possibly nonempty boundary into an open domain $\mathcal{D}$ contained in a three-manifold $N$ with possibly nonempty boundary. The limit set of $M$ is

$$
L(M)=\bigcap_{\alpha \in I}\left(\overline{f(M)-f\left(E_{\alpha}\right)}\right)
$$

where $\left\{E_{\alpha}\right\}_{\alpha \in I}$ is the collection of compact subdomains of $M$ and the closure $\overline{f(M)-f\left(E_{\alpha}\right)}$ is taken in $N$. The limit set $L(e)$ of an end $e$ of $M$ is defined to be the intersection of the limit sets all properly embedded subdomains of $M$ with compact boundary which represent e. Notice that $L(M)$ and $L(e)$ are closed sets of $\partial \mathcal{D}$, and so each of these limit sets is compact when $N$ is compact. 
First we will prove Theorem 1.1 in the case $\bar{W}$ is the smooth closed Riemannian ball $\overline{\mathbb{B}} \subset \mathbb{R}^{3}$. In this case, we will construct a properly embedded 1-manifold $\Delta \subset \mathbb{B}$ with path components consisting of smooth simple closed curves such that every proper immersion $f: A=\mathbb{S}^{1} \times[0, \infty) \rightarrow \mathbb{B}-$ $\Delta$ of an annulus with a complete induced metric has unbounded mean curvature; this result will prove Theorem 1.1 in the special case $\bar{W}=\overline{\mathbb{B}}$. The proof of the case of Theorem 1.1 when $\bar{W}$ is a smooth Riemannian ball, or more generally, an arbitrary compact smooth Riemannian manifold with nonempty boundary follows from straightforward modifications of the proof of the $\mathbb{B}-\Delta$ case; these modifications are outlined in the last paragraph of the proof.

The first step in the construction of $\Delta$ is to create a $C W$-complex structure $\Lambda$ on the open ball $\mathbb{B}$. Consider the boundary $\partial$ of the box $[-1,1] \times[-1,1] \times[-1,1] \subset \mathbb{R}^{3}$. The surface $\partial$ has a natural structure of a simplicial complex $\mathcal{X}_{1}$ with faces $\mathcal{F}_{1}=\left\{F_{1}, F_{2}, \ldots, F_{6}\right\}$ contained in planes parallel to the coordinate planes, edges $\mathcal{E}_{1}=\left\{E_{1}, E_{2}, \ldots, E_{12}\right\}$ and vertices $\mathcal{V}_{1}=\left\{v_{1}, v_{2}, \ldots, v_{8}\right\}$. Let $\mathcal{X}_{2}$ denote the related refined simplicial complex obtained from $\mathcal{X}_{1}$ by adding vertices to the centers of each of the faces of $\mathcal{F}_{1}$ and to the centers of each of the edges in $\mathcal{E}_{1}$, thereby obtaining new collections $\mathcal{F}_{2}, \mathcal{E}_{2}, \mathcal{V}_{2}$ of faces, edges, and vertices. In this subdivision each face of $\mathcal{F}_{2}$ corresponds to subsquare in one the faces in $\mathcal{F}_{1}$ with four line segments, each of length one. Note that $\mathcal{F}_{2}$ has $6 \cdot 4$ faces, $\mathcal{E}_{2}$ has $2 \cdot 6 \cdot 4$ edges and $\mathcal{V}_{2}$ has $6 \cdot 4+2$ vertices. Continuing inductively the refining of the complex $\mathcal{X}_{2}$, produces at the $n$-th stage a simplicial complex $\mathcal{X}_{n}$ with $6 \cdot 4^{n-1}$ square faces $\mathcal{F}_{n}, 2 \cdot 6 \cdot 4^{n-1}$ edges $\mathcal{E}_{n}$ and $6 \cdot 4^{n-1}+2$ vertices $\mathcal{V}_{n}$.

We define the 1-skeleton $\Gamma$ of $\Lambda$ as follows:

$$
\Gamma=\bigcup_{k=1}^{\infty}\left[C\left(\mathcal{E}_{k}\right) \cap \mathbb{S}_{k}^{2}\right] \cup\left[C\left(\mathcal{V}_{k}\right) \cap\left(\overline{\mathbb{B}}_{k+1}-\mathbb{B}_{k}\right)\right]
$$

where $C\left(\mathcal{E}_{k}\right)$ denotes the cone $C\left(\cup \mathcal{E}_{k}\right)$. Extend the proper 1-dimensional $C W$-complex $\Gamma \subset \mathbb{B}$ to a proper 2-dimensional $C W$-subcomplex $\Lambda^{\prime}$ of $\Lambda$ as follows. The faces of $\Lambda^{\prime}$ are the spherical squares in $\mathbb{S}_{k}^{2}-\Gamma$, as $k$ varies in $\mathbb{N}$, together with the set of flat rectangles $C(\alpha) \cap\left(\mathbb{B}_{k+1}-\overline{\mathbb{B}}_{k}\right)$, where $\alpha$ is a 1 -simplex in $\Gamma \cap \mathbb{S}_{k}^{2}$, as $k$ varies in $\mathbb{N}$ and $\alpha$ varies in $\Gamma \cap \mathbb{S}_{k}^{2}$, see Figure 1 below. Let $\mathcal{F}$ denote the set of faces of $\Lambda$. Finally, $\mathbb{B}-\Lambda^{\prime}$ contains an infinite collection $\mathcal{G}=\left\{G_{\alpha}\right\}_{\alpha \in I}$ of components which have the appearance of a cube which is a radial product of a spherical square in some $\mathbb{S}_{k}^{2}-\Gamma$ with a small interval of length $2^{-(k+1)}$, together with the special component $\mathbb{B}\left(\frac{1}{2}\right)$. The set $\mathcal{G}$ is the set of 3 -cells in $\Lambda$, which completes the construction of the $C W$-complex structure $\Lambda$ of $\mathbb{B}$.

Define the related closed, piecewise smooth regular neighborhood $\widehat{N}(\Gamma)$ of $\Gamma$ :

$$
\widehat{N}(\Gamma)=\bigcup_{k=1}^{\infty}\left[\left(C\left(\mathcal{E}_{k}\right) \cap \mathbb{S}_{k}^{2}\right)\left(\frac{1}{2^{k} 10}\right)\right] \cup\left[\left(C\left(\mathcal{V}_{k}\right) \cap\left(\overline{\mathbb{B}}_{k+1}-\mathbb{B}_{k}\right)\right)\left(\frac{1}{2^{k} 100}\right)\right] .
$$

Then let $N(\Gamma) \subset \operatorname{Int}(\widehat{N}(\Gamma))$ be a small smooth closed regular neighborhood of $\Gamma$ in $\mathbb{B}$ such that its boundary $\partial N(\Gamma)$ intersects each face $F$ in $\mathcal{F}$ transversely in a simple closed curve $\beta(F)$ that bounds a disk $L(F) \subset F$; let $\mathcal{L}=\{L(F) \mid F \in \mathcal{F}\}$. For each open 1-simplex $\alpha \in \Gamma$, let $P(\alpha)$ be the plane perpendicular to $\alpha$ at the midpoint of $\alpha$. Let $\widetilde{N}(\Gamma) \subset \operatorname{Int}(\widehat{N}(\Gamma))$ be another smooth closed regular neighborhood of $\Gamma$ with $N(\Gamma) \subset \operatorname{Int}(\widetilde{N}(\Gamma))$ and such that $\partial \widetilde{N}(\Gamma) \cap P(\alpha)$ contains a simple closed curve $\beta(\alpha)$ close to $\alpha$ and which links $\alpha$. Let $W(\alpha) \subset P(\alpha)$ denote the closed disk with boundary curve $\beta(\alpha)$ and let $\mathcal{W}=\{W(\alpha) \mid \alpha \in \Gamma\}$, see Figure 2 .

The set $\Delta$ is the collection $\left[\bigcup_{\alpha \in \Gamma} \beta(\alpha)\right] \cup\left[\bigcup_{F \in \mathcal{F}} \beta(F)\right]$. The domain described in Theorem 1.1 is $\mathcal{D}=\mathbb{B}-\Delta$.

We conclude this section with the following immediate consequence of our constructions above. 


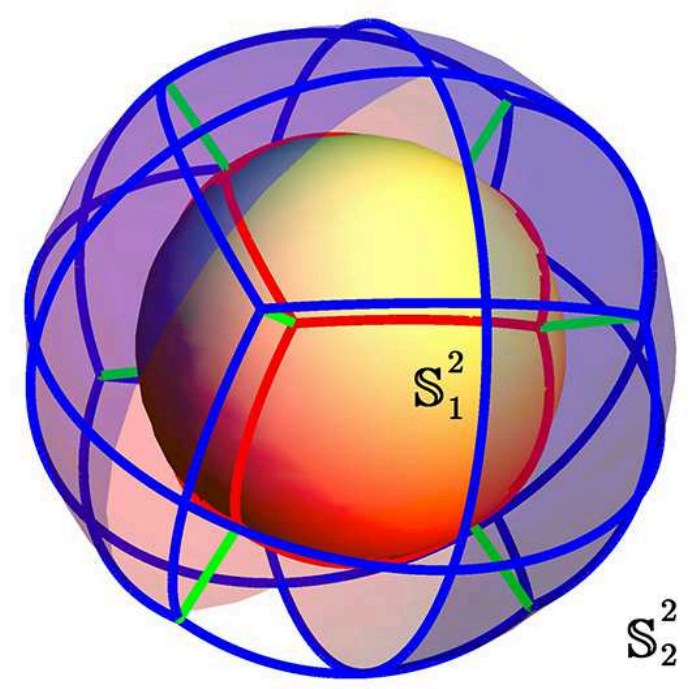

Figure 1: The first two steps in the construction of $\Lambda$.

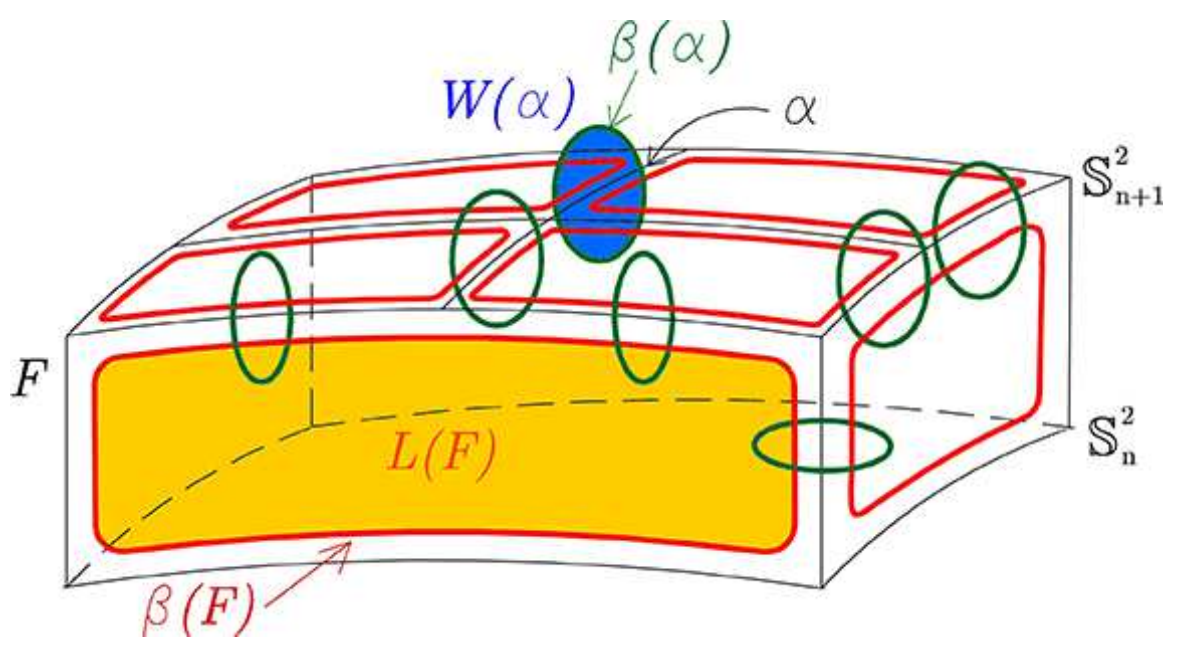

Figure 2: The 1-dimensional simplicial complex $\Gamma$, the 1-manifold $\Delta$ consisting of closed curves $\beta(F)$ and $\beta(F)$ and the disks $W(\alpha)$ and $L(F)$, where $F$ is a face in $\Lambda$ and $\alpha$ is a 1-simplex in $\Lambda$. 
Lemma 2.2 Let $E$ be one of the following:

1. a 1-simplex, face or 3-cell in $\Lambda$;

2. a disk in either $\mathcal{W}$ or $\mathcal{L}$;

3. a component of $\tilde{N}(\Gamma)-\cup \mathcal{W}$.

If for some $\delta \in\left(0, \frac{1}{4}\right), E \cap[\mathbb{B}-\mathbb{B}(1-\delta)] \neq \varnothing$, then $E$ is contained in a ambient ball $B_{E}$ of radius $4 \delta$.

\section{$3 L(A)$ is a path connected subset of $\mathbb{S}^{2}$ with more than one point.}

In this and the following sections, $f: A \rightarrow \mathcal{D}$ will denote a counterexample to Theorem 1.1 which, after a small smooth perturbation, we will assume to be a fixed properly immersed annulus diffeomorphic to $\mathbb{S}^{1} \times[0,1)$ satisfying:

1. The supremum of the absolute mean curvature of $A$ is less than a fixed constant $H_{0}>10$;

2. $f$ is transverse to the disks in $\mathcal{W}$ and to the surface $\partial \widetilde{N}(\Gamma)$;

3. $f$ is in general position with respect to $\Lambda$, i.e., $f$ is disjoint from the set of vertices $\mathcal{V}$ of $\Lambda$, transverse to the closed faces of $\Lambda$ and so, it is also transverse to $\mathbb{S}_{k}^{2}$ for each $k \in \mathbb{N}$.

Lemma 3.1 If $f: \Sigma \rightarrow \mathcal{D}$ is a properly immersed surface with compact boundary and $\mathbf{e}$ is an end of $\Sigma$, then the limit set $L(\mathbf{e})$ of the end $\mathbf{e}$ is path connected.

Proof. This is a standard result, but for the sake of completeness, we present its proof. Let $p, q \in L(\mathbf{e})$ be distinct points. Let $\mathcal{D}_{1} \subset \mathcal{D}_{2} \subset \ldots \subset \mathcal{D}_{n} \subset \ldots$ be a smooth compact exhaustion of $\mathcal{D}$. After replacing by subsequences, we may assume that there is a sequence of pairs of points $p_{n}, q_{n}$ which lie in the component of $\Sigma-\operatorname{Int}\left(f^{-1}\left(\mathcal{D}_{n}\right)\right)$ which represents e and such that $\lim _{n \rightarrow \infty} f\left(p_{n}\right)=p$ and $\lim _{n \rightarrow \infty} f\left(q_{n}\right)=q$.

Let $\sigma_{n}:[0,1] \rightarrow \Sigma-\operatorname{Int}\left(f^{-1}\left(\mathcal{D}_{n}\right)\right)$ be paths with $\sigma_{n}(0)=p_{n}$ and $\sigma_{n}(1)=q_{n}$. Since the space $\mathcal{C}([0,1], \overline{\mathbb{B}})$ of continuous maps of $[0,1]$ into $\overline{\mathbb{B}}$ is a compact metric space in the sup norm, a subsequence of the paths $f \circ \sigma_{n}$ converges to a continuous map $f \circ \sigma$ of $[0,1]$ to $\partial \mathcal{D}=\left[\mathbb{S}^{2} \cup \Delta\right] \subset \overline{\mathbb{B}}$ with $f \circ \sigma(0)=p$ and $f \circ \sigma(1)=q$. Since $f \circ \sigma([0,1]) \subset L(\mathbf{e})$ also holds, $L(\mathbf{e})$ is path connected.

Lemma 3.2 If $L(A) \cap \Delta \neq \varnothing$ or if $L(A)$ consists of a single point in $\mathbb{S}^{2}$, then $A$ has finite area.

Proof. By Theorems 3.1 and 3.1' in [3], the bounded mean curvature hypothesis and the properness hypothesis on $f$ imply that if $f$ composed with the inclusion map of $\mathcal{D}$ into $\mathbb{R}^{3}$ is proper outside of a point in $\mathbb{S}^{2}$ or outside of a component of $\Delta$, then the surface $A$ has finite area. Since $L(A)$ is path connected and the path components of $\partial \mathcal{D}$ are $\mathbb{S}^{2}$ or a simple closed curve in $\Delta$, then the lemma follows.

Lemma 3.3 If $F: A \rightarrow \mathbb{R}^{3}$ is a complete immersion of $\mathbb{S}^{1} \times[0, \infty)$ with bounded mean curvature, then $A$ has infinite area. 
Proof. Suppose that $A$ has finite area and we will obtain a contradiction. Since $A$ is a complete annulus of finite area, there exists a sequence $\gamma_{n}$ of pairwise disjoint, piecewise smooth, closed embedded geodesics with a single corner, which are topologically parallel to $\partial A$ and whose lengths tend to 0 as $n$ tends to infinity. Assume that the index ordering of the geodesics $\gamma_{n}$ agrees with the relative distances of these curves to $\partial A$. Replace $A$ by the subend $A\left(\gamma_{1}\right)$ with $\partial A\left(\gamma_{1}\right)=\gamma_{1}$. By the Gauss-Bonnet formula applied to the subannulus $A\left(\gamma_{1}, \gamma_{n}\right)$ with boundary $\gamma_{1} \cup \gamma_{n}$, the total Gaussian curvature of $A\left(\gamma_{1}, \gamma_{n}\right)$ is greater than $-4 \pi$. Since the Gaussian curvature function $K_{A}$ of $A$ is pointwise bounded from above by $H_{0}^{2}$, then the integral $\int_{A\left(\gamma_{1}\right)} K_{A}^{+} d A$, where $K_{A}^{+}(x)=\max \left\{K_{A}(x), 0\right\}$, is finite because $A$ has finite area. Hence, after replacing $A$ by a subend of $A$, we may assume that $\int_{A\left(\gamma_{1}\right)} K_{A}^{+} d A<\pi$. So, we conclude that $\int_{A\left(\gamma_{1}, \gamma_{n}\right)} K_{A}^{-} d A>-5 \pi$, for all $n$, where $K_{A}^{-}(x)=$ $\min \left\{K_{A}(x), 0\right\}$.

On the other hand, since the area of $A$ does not grow at least linearly with the distance from $\partial A$, the norm of the second fundamental form of $A$ is unbounded on $A$. By standard rescaling arguments (see for example [5]), there exists a divergent sequence $p_{n} \in A\left(\gamma_{1}\right)$ of blow-up points on the scale of the second fundamental form with norm of the second fundamental form at $p_{n}$ being $\lambda_{n}>n$, and intrinsic neighborhoods $B_{A}\left(p_{n}, \frac{\lambda_{n}}{10}\right)$ such that a subsequence of the rescaled surfaces $\lambda_{n}\left[f\left(B_{A}\left(p_{n}, \frac{\lambda_{n}}{10}\right)\right)-p_{n}\right]$ converges in the $C^{2}$-norm to a minimal disk $D$ in $\mathbb{R}^{3}$ satisfying:

1. The norm of the second fundamental form of $D$ is at most 1 and equal to 1 at the origin.

2. $D$ is a graph over the projection to its tangent plane at the origin.

3. The total curvature of $D$ is $-\varepsilon$ for some $\varepsilon>0$. Hence for $n$ large, the integral of the function $K_{A}^{-}$on $B_{A}\left(p_{n}, \frac{\lambda_{n}}{10}\right)$ is less than $-\frac{\varepsilon}{2}$.

By property 3 above, we conclude that $\lim _{n \rightarrow \infty} \int_{A\left(\gamma_{1}, \gamma_{n}\right)} K_{A}^{-} d A=-\infty$, which contradicts our earlier observation that $\int_{A\left(\gamma_{1}, \gamma_{n}\right)} K_{A}^{-} d A$ is bounded from below by $-5 \pi$.

The next lemma is an immediate consequence of Lemmas 3.2 and 3.3

Lemma 3.4 $L(A)$ is a path connected compact subset of $\mathbb{S}^{2}$ containing two distinct points $x$ and $y$. In particular, the immersion $f$ can be seen as a proper immersion in $\mathbb{B}$.

In the next sections, we will analyze how certain subdomains of the immersed annulus $f(A)$ intersects certain specific two-dimensional subsets of $\mathcal{D}$, for which we need the following definitions.

Definition 3.5 Suppose $F: \Sigma \rightarrow \mathcal{D}$ is a smooth proper immersion of a surface with compact boundary which is transverse to the disks in $\mathcal{W}$, to $\partial \widetilde{N}(\Gamma)$ and is in general position with respect to $\Lambda$. Suppose $\gamma$ is a simple closed curve in $\Sigma$. Then:

1. $\gamma$ is an $X_{1}$-type curve, if $\gamma$ is a component of $F^{-1}(\cup \mathcal{W})$.

2. $\gamma$ is an $X_{2}$-type curve, if $\gamma$ is a component of $F^{-1}(\partial \widetilde{N}(\Gamma))$. Note that in this case $\gamma \subset$ $[\partial \widetilde{N}(\Gamma)-\cup \mathcal{W}]$ and so curves of $X_{1}$-type and $X_{2}$-type are disjoint.

3. $\gamma \subset \Sigma$ is an $X_{3}$-type curve, if $\gamma$ is a component of $F^{-1}(\cup \mathcal{L})$. Notice that in this case $\gamma$ is contained in a face of $\Lambda$.

Definition 3.6 Given the fixed immersion $f: A \rightarrow \mathcal{D}$, then: 
1. $X_{1}$ is the set of $X_{1}$-type curves parallel to $\partial A$ and $X_{2}$ is the set of $X_{2}$-type curves parallel to $\partial A$.

2. $X_{3}$ is the set of $X_{3}$-type curves in $A$ which are disjoint from $\left(\cup X_{1}\right) \cup\left(\cup X_{2}\right)$.

3. By Lemma 4.1 below, the countable set $X$ can be expressed as $X=X_{1} \cup X_{2} \cup X_{3}=$ $\left\{\gamma_{i} \mid i \in \mathbb{N}\right\}$, where the natural ordering of the simple closed, pairwise-disjoint curves $\gamma_{i}$ in $A$ by their relative distances from $\partial A$ agrees with the ordering of the index set $\mathbb{N}$.

4. $A_{n}$ denotes the compact subannulus in $A$ with $\partial A_{n}=\partial A \cup \gamma_{n}$; note $A_{1} \subset A_{2} \subset \ldots \subset A_{n} \subset$ ... is a smooth compact exhaustion of $A$.

5. For $n, j \in \mathbb{N}, A(n, j)$ denotes the compact subannulus of $A$ with boundary curves $\gamma_{n}$ and $\gamma_{n+j}$.

6. $A(k)=\cup_{j=1}^{\infty} A(k, j)$ is the end representative of $A$ with boundary $\gamma_{k}$.

\section{Placement properties of $\partial A(k, 1)$ for $\mathbf{k}$ large.}

Lemma 4.1 For $k$ large, there exists at least one curve in $X$ in the region $\mathbb{B}_{k+2}-\overline{\mathbb{B}}_{k-1}$. In particular, the set $X$ is infinite.

Proof. Assume that $f(\partial A)$ is contained in $\mathbb{B}_{n}$ and we will prove that $\mathbb{B}_{k+2}-\overline{\mathbb{B}}_{k-1}$ contains an element in $X$, whenever $k>n$. Since $f: A \rightarrow \mathbb{B}$ is proper and transverse to the spheres $\mathbb{S}_{i}^{2}$ for every $i$, then for $i \geq n, f^{-1}\left(\mathbb{S}_{i}^{2}\right)$ contains a simple closed curve $\alpha_{i}$ which is a parallel to $\partial A$. If $f\left(\alpha_{k}\right) \cap(\cup \mathcal{L}) \neq$ $\varnothing$, then either $\alpha_{k} \in X_{3}$ or $\alpha_{k}$ intersects an element $\gamma$ of $X_{1} \cup X_{2}$, where $f(\gamma)$ is contained in $\left[\mathbb{B}_{k+1}-\overline{\mathbb{B}}_{k-1}\right] \subset\left[\mathbb{B}_{k+2}-\overline{\mathbb{B}}_{k-1}\right]$. Similarly, if $f\left(\alpha_{k+1}\right) \cap(\cup \mathcal{L}) \neq \varnothing$, then either $\alpha_{k+1} \in X_{3}$ or $\alpha_{k+1}$ intersects an element $\gamma$ of $X_{1} \cup X_{2}$, whose image $f(\gamma)$ must be contained in $\left[\mathbb{B}_{k+2}-\overline{\mathbb{B}}_{k}\right] \subset$ $\left[\mathbb{B}_{k+2}-\overline{\mathbb{B}}_{k-1}\right]$. Hence, we may assume that $f\left(\alpha_{k}\right)$ and $f\left(\alpha_{k+1}\right)$ are both disjoint from $\cup \mathcal{L}$ and so, $\left[f\left(\alpha_{k} \cup \alpha_{k+1}\right)\right] \subset \operatorname{Int}(\widetilde{N}(\Gamma))$.

Let $D_{\mathcal{W}}^{k}$ be the collection of disks in $\mathcal{W}$ which are contained in $\mathbb{B}_{k+1}-\overline{\mathbb{B}}_{k}$ and let $\Sigma^{k}$ be the compact domain which is closure of the component of $\widetilde{N}(\Gamma)-\left(\cup D_{\mathcal{W}}^{k}\right)$ which contains $f\left(\alpha_{k}\right)$ in its interior. Let $A\left(\alpha_{k}, \alpha_{k+1}\right)$ be the subannulus of $A$ with boundary $\alpha_{k} \cup \alpha_{k+1}$. Then $\left(\left.f\right|_{A\left(\alpha_{k}, \alpha_{k+1}\right)}\right)^{-1}\left(\partial \Sigma^{k}\right)$ contains a simple closed curve $\gamma$ which is parallel to $\partial A$ and which is an element of $X_{1} \cup X_{2} \subset X$. The existence of $\gamma$ completes the proof of the assertion.

Lemma 4.2 There exists a small $\eta_{1}>0$ such that for any $\eta \in\left(0, \eta_{1}\right]$, if $D \subset A$ is a compact disk with $f(\partial D) \subset \mathbb{B}(z, \eta)$ for some $z \in \mathbb{S}^{2}$ and $D$ contains a point $p$ such that the distance $d(f(p), z) \geq 1$, then:

1. The disk $D$ contains a $X_{i}$-type curve $\beta$, for $i=1,2$ or 3 , and $f(\beta)$ lies in $\mathbb{B}(z, 1 / 2)-\overline{\mathbb{B}}(z, 2 \eta)$.

2. The curve $\beta$ can be chosen so that the disk $D(\beta) \subset D$ bounded by $\beta$ contains $p$. In particular, $f(D(\beta))$ contains a point of distance at least $\frac{1}{2}$ from its boundary and every point in $D(\beta)$ has intrinsic distance at least $\eta$ from $\partial D$.

Proof. Recall that for any face $F$ in $\mathcal{F}, C(F)$ denotes the cone over $F$. Clearly, for $\eta_{1}>0$ sufficiently small and $\eta \in\left(0, \eta_{1}\right]$, there exist faces $F_{1}, F_{2}, F_{3}$ and $F_{4}$ in $\mathcal{F}$, such that: $\mathbb{B}(z, 2 \eta) \subset \operatorname{Int}\left(C\left(F_{1}\right)\right)$, $C\left(F_{i}\right) \subset \operatorname{Int}\left(C\left(F_{i+1}\right)\right)$, for $i=1,2,3$ and $C\left(F_{4}\right) \subset \mathbb{B}(z, 1 / 2)$. 
At this point we can follow the proof of Lemma 4.1 where the annulus $D-\{p\}$ plays the role of $A$ and the piecewise smooth disk $\partial C\left(F_{i}\right)$ plays the role of $\mathbb{S}_{k-2+i}^{2}$. Then we obtain an $X_{i}$-type curve $\beta$ parallel to $\partial D$ in $D-\{p\}$ and whose image $f(\beta)$ is in the open region between $\partial\left(C\left(F_{1}\right)\right)$ and $\partial\left(C\left(F_{4}\right)\right)$, which is contained $\mathbb{B}(z, 1 / 2)-\overline{\mathbb{B}}(z, 2 \eta)$. This is the desired curve.

Before stating the next assertion, we need some notation.

Definition 4.3 Given a curve $\gamma_{k}$ in $X$, we define $\chi_{1}\left(f\left(\gamma_{k}\right)\right)$ to be the union of all closed 3-cells in $\Lambda$ which intersect $f\left(\gamma_{k}\right)$. Similarly, given $i \in \mathbb{N}$ we define $\chi_{i+1}\left(f\left(\gamma_{k}\right)\right)$ as the union of all closed 3-cells in $\Lambda$ which intersect $\chi_{i}\left(f\left(\gamma_{k}\right)\right)$.

In what follows, we shall use the observation that for $i=1$ and 2 , the set $\chi_{i}\left(f\left(\gamma_{k}\right)\right)$ is a piecewise smooth compact ball, whose boundary sphere is a union of faces in $\mathcal{F}$ and it is in general position with respect to the immersion $f$.

Lemma 4.4 For $k$ large, we have $f\left(\gamma_{k+1}\right) \subset \chi_{3}\left(f\left(\gamma_{k}\right)\right)$ or $f\left(\gamma_{k}\right) \subset \chi_{3}\left(f\left(\gamma_{k+1}\right)\right)$. Furthermore, given $\eta>0$, there exists an integer $k(\eta)$ such that for any $k \geq k(\eta)$ one has:

1. $f(A(k)) \subset[\mathbb{B}-\overline{\mathbb{B}}(1-\eta)]$ and each $X_{i}$-type curve $\gamma, i=1,2$ or 3 , in $A(k, 1)$ is contained in a ball $\mathbb{B}(y(\gamma), \eta)$ for a suitable point $y(\gamma) \in \mathbb{S}^{2}$.

2. There is a point $z(k) \in \mathbb{S}^{2}$ such that $f\left(\gamma_{k} \cup \gamma_{k+1}\right) \subset \mathbb{B}(z(k), \eta)$.

3. Every simple closed curve $\gamma \subset\left[A(k, 1)-f^{-1}(\mathbb{B}(z(k), \eta))\right]$ bounds a disk in $A(k, 1)$.

Proof. In order to prove the first statement of the lemma, we distinguish four cases, depending on the position of $f\left(\gamma_{k}\right)$. We will use the fact that by Lemma 2.2 for $k \rightarrow \infty$, the curve $f\left(\gamma_{k}\right)$ becomes arbitrarily close to a point $z(k) \in \mathbb{S}^{2}$.

Case A: $f\left(\gamma_{k}\right) \subset D \in \mathcal{W}$.

In this case $f(A(k, 1))$ enters a component $C$ of $\widetilde{N}-\cup \mathcal{W}$ near $f\left(\gamma_{k}\right)$. Consider the compact component $Z$ of $\left(\left.f\right|_{A(k)}\right)^{-1}(\bar{C}) \subset A(k)$ with boundary component $\gamma_{k}$ and let $\alpha_{k}$ be the boundary curve of $Z-\gamma_{k}$ which is parallel to $\partial A(k)=\gamma_{k}$. By the definition of $X_{1}$ and $X_{2}, \alpha_{k}=\gamma_{k+j} \in$ $\left[X_{1} \cup X_{2}\right] \subset X$, for some $j \geq 1$. By definition of $X, \gamma_{k+1} \subset A(k, j)$ and intersects the domain $Z$. If $\gamma_{k+1} \subset Z$, then clearly $f\left(\gamma_{k+1}\right) \subset \bar{C} \subset \chi_{2}\left(f\left(\gamma_{k}\right)\right)$ and we are done. Otherwise, $f\left(\gamma_{k+1}\right)$ must not be contained in $\widetilde{N}(\Gamma)$. This means that $\gamma_{k+1}$ belongs to $X_{3}$ and so it is contained in a face $F$ of $\Lambda$, which intersects $C$. Hence, $F \subset \chi_{2}\left(f\left(\gamma_{k}\right)\right)$ which implies $f\left(\gamma_{k+1}\right) \subset \chi_{2}\left(f\left(\gamma_{k}\right)\right)$.

Case B: $f\left(\gamma_{k}\right) \subset[\partial \widetilde{N}(\Gamma)-\cup \mathcal{W}]$ and the annulus $f(A(k, 1))$ enters $\widetilde{N}(\Gamma)$ near $f\left(\gamma_{k}\right)$.

In this case, the arguments in Case A apply to show that $f\left(\gamma_{k+1}\right) \subset \chi_{2}\left(f\left(\gamma_{k}\right)\right)$.

Case C: $f\left(\gamma_{k}\right) \subset[\partial \widetilde{N}(\Gamma)-\cup \mathcal{W}]$ and the annulus $f(A(k, 1))$ enters $\mathbb{B}-\widetilde{N}(\Gamma)$ near $f\left(\gamma_{k}\right)$.

First, note that if $f\left(\gamma_{k+1}\right)$ intersects $\chi_{2}\left(f\left(\gamma_{k}\right)\right)$, then $f\left(\gamma_{k+1}\right) \subset \chi_{3}\left(f\left(\gamma_{k}\right)\right)$. Thus, we may assume that $f\left(\gamma_{k+1}\right)$ lies outside the compact piecewise smooth ball $\chi_{2}\left(f\left(\gamma_{k}\right)\right)$. Consider the compact component $Z$ of $\left(\left.f\right|_{A(k, 1)}\right)^{-1}\left(\chi_{2}\left(f\left(\gamma_{k}\right)\right)\right)$ containing $\gamma_{k}$ in its boundary. Let $\alpha_{k} \neq \gamma_{k}$ be the boundary curve of $Z$ which is parallel in $A(k)$ to $\gamma_{k}$; recall that $A(k)$ is the end of $A$ with boundary $\gamma_{k}$. If $f\left(\alpha_{k}\right)$ intersects $\cup \mathcal{L}$, then $f\left(\alpha_{k}\right)$ is contained in a disk $D \in \mathcal{L}$; in this case, since $\alpha_{k}$ lies between $\gamma_{k}$ and $\gamma_{k+1}$ and it is parallel to $\partial A(k)$, then $\alpha_{k} \in X_{3}$, which is contrary to the definition of $\gamma_{k+1}$. Thus, $f\left(\alpha_{k}\right) \subset \partial\left(\chi_{2}\left(f\left(\gamma_{k}\right)\right)\right)$ and is disjoint from $\cup \mathcal{L}$, and so $f\left(\alpha_{k}\right) \subset \operatorname{Int}(\widetilde{N}(\Gamma))$. Let $A\left(\gamma_{k}, \alpha_{k}\right) \subset A(k, 1)$ be the subannulus with boundary curves $\gamma_{k} \cup \alpha_{k}$. As $f\left(A\left(\gamma_{k}, \alpha_{k}\right)\right)$ enters 
$\mathbb{B}-\tilde{N}(\Gamma)$ nears $f\left(\gamma_{k}\right)$ and $f\left(\alpha_{k}\right) \subset \operatorname{Int}(\tilde{N}(\Gamma))$, then ours previous separation arguments imply that there exists a curve $\beta \subset\left(\left.f\right|_{A\left(\gamma_{k}, \alpha_{k}\right)}\right)^{-1}(\partial \widetilde{N}(\Gamma)-\cup \mathcal{W})$ which is parallel to $\gamma_{k}$. Since $\beta \in X_{2}$ and $\beta \neq \gamma_{k+1}$, we arrive at a contradiction. This contradiction proves Case C.

Case D: $f\left(\gamma_{k}\right) \subset D \in \mathcal{L}$.

If $f\left(\gamma_{k+1}\right) \subset \partial \widetilde{N}(\Gamma)$ or $f\left(\gamma_{k+1}\right) \subset \widehat{D} \in \mathcal{W}$, then the arguments in our previously considered cases imply that $f\left(\gamma_{k}\right) \subset \chi_{3}\left(f\left(\gamma_{k+1}\right)\right)$. Hence, we may assume that $f\left(\gamma_{k+1}\right) \subset D^{\prime} \in \mathcal{L}$ as well.

If $\chi_{1}\left(f\left(\gamma_{k}\right)\right) \cap \chi_{1}\left(f\left(\gamma_{k+1}\right)\right) \neq \varnothing$, then $f\left(\gamma_{k+1}\right) \subset \chi_{2}\left(f\left(\gamma_{k}\right)\right)$. Hence, we can assume that $\chi_{1}\left(f\left(\gamma_{k}\right)\right) \cap \chi_{1}\left(f\left(\gamma_{k+1}\right)\right)=\varnothing$. Recall that $\left.f\right|_{A(k, 1)}$ is in general position with respect to $\partial\left(\chi_{1}\left(f\left(\gamma_{k}\right)\right)\right)$ and $\partial\left(\chi_{1}\left(f\left(\gamma_{k+1}\right)\right)\right)$. Let $Z_{i}$ be the component of $\left(\left.f\right|_{A(k, 1)}\right)^{-1}\left(\chi_{1}\left(f\left(\gamma_{i}\right)\right)\right)$ with boundary component $\gamma_{i}$ and let $\alpha_{i} \neq \gamma_{i}$ be the boundary component of $Z_{i}$ which is parallel to $\gamma_{i}$, for $i=k, k+1$, respectively. Since $\alpha_{k}$ and $\alpha_{k+1}$ lie in $\operatorname{Int}(A(k, 1))$, then by definition of $X$, both $f\left(\alpha_{k}\right)$ and $f\left(\alpha_{k+1}\right)$ are disjoint from $\cup \mathcal{L}$. Moreover, as $f\left(\alpha_{i}\right) \subset \chi_{1}\left(f\left(\gamma_{i}\right)\right)$, for $i=k, k+1$, then $f\left(\alpha_{k} \cup \alpha_{k+1}\right) \subset$ $\operatorname{Int}(\tilde{N}(\Gamma))$. Let $A\left(\alpha_{k}, \alpha_{k+1}\right)$ be the subannulus of $A(k, 1)$ with boundary $\alpha_{k} \cup \alpha_{k+1}$.

Consider the collection of disks $D_{\mathcal{W}}^{k}$ in $\mathcal{W}$ which are contained in the interior of $\chi_{2}\left(f\left(\gamma_{k}\right)\right)-$ $\chi_{1}\left(f\left(\gamma_{k}\right)\right)$. Then $\tilde{N}(\Gamma)-\cup D_{\mathcal{W}}^{k}$ contains a connected domain whose closure $\Sigma^{k}$ in $\mathbb{B}$ satisfies $f\left(\alpha_{k}\right) \subset$ $\operatorname{Int}\left(\Sigma^{k}\right)$ and $f\left(\alpha_{k+1}\right) \subset \mathbb{B}-\Sigma^{k}$. Our previous separation arguments imply that there is a simple closed curve $\beta$ in $\left(\left.f\right|_{A\left(\alpha_{k}, \alpha_{k+1}\right)}\right)^{-1}\left(\partial \Sigma^{k}\right)$ which is parallel to $\gamma_{k}$. But $\beta \subset \operatorname{Int}\left(A(k, 1)\right.$ and $\beta \in X_{1} \cup X_{2}$, which is a contradiction. This contradiction completes the proof of the first statement of the lemma.

Item 1 in the lemma is a straightforward consequence of the fact that, as $\rightarrow \infty$, then $f(A(k))$ uniformly converges to $\mathbb{S}^{2}$. Moreover, given a $X_{i}$-type curve $\gamma \subset A(k), i=1,2,3$, the Euclidean diameter of $f(\gamma)$ goes to zero (as $k \rightarrow \infty$ ) and is arbitrarily close to a point $y(\gamma)$ in $\mathbb{S}^{2}$. Item 2 in the lemma follows from the observation that as $k \rightarrow \infty$, the sets $\chi_{3}\left(f\left(\gamma_{k}\right)\right)$ are arbitrarily close to $f\left(\gamma_{k}\right)$, which in turn, lie arbitrarily close to points $z(k) \in \mathbb{S}^{2}$. These observations imply that there exists an integer $j(\eta)$ such that for $k \geq j(\eta)$, items 1 and 2 in Lemma4.4 hold.

In order to obtain item 3 , we define $k(\eta)=j\left(\frac{\eta}{900}\right)$. By definition of $j\left(\frac{\eta}{900}\right)$, for $k \geq k(\eta)$, $f\left(\gamma_{k} \cup \gamma_{k+1}\right) \subset \mathbb{B}\left(z(k), \frac{\eta}{900}\right)$ and $f(A(k)) \subset\left[\mathbb{B}-\overline{\mathbb{B}}\left(1-\frac{\eta}{900}\right)\right]$. It remains to check that each simple closed curve $\beta$ in a component $K$ of $\left(f_{A(k, 1)}\right)^{-1}(\mathbb{B}-\mathbb{B}(z(k), \eta))$ bounds a disk in $A(k, 1)$; note that

$\bar{K} \subset \operatorname{Int}(A(k, 1))$. Observe that $\frac{\eta}{900}$ is sufficiently small so that there exist faces $F_{1}, F_{2}, F_{3}$ and $F_{4}$ in $\mathcal{F}$, such that: $\mathbb{B}\left(z(k), \frac{\eta}{900}\right) \subset \operatorname{Int}\left(C\left(F_{1}\right)\right), C\left(F_{i}\right) \subset \operatorname{Int}\left(F_{i+1}\right)$, for $i=1,2,3$ and $C\left(F_{4}\right) \subset$ $\mathbb{B}(z(k), \eta)$.

If $\beta \subset K$ does not bound a disk in $A(k, 1)$, then it is parallel to $\gamma_{k}$ in $A(k, 1)$. Let $A\left(\gamma_{k}, \beta\right)$ denote the subannulus of $A(k, 1)$ with boundary $\gamma_{k} \cup \beta$. Then the arguments in the proof of Lemma4.2 imply that there exists a simple closed curve $\gamma^{\prime} \subset \operatorname{Int}\left(A\left(\gamma_{k}, \beta\right)\right)$ which is parallel to $\gamma_{k}, f\left(\gamma^{\prime}\right) \subset \mathbb{B}(z(k), \eta)$ and $\gamma^{\prime}$ is an $X_{i}$-type curve, for $i=1,2$ or 3 . In particular, $\gamma^{\prime} \in X$ which is impossible. Thus, every simple closed curve in $A(k, 1)$ whose image under $f$ lies outside of $\mathbb{B}(z(k), \eta)$ bounds a disk in $A(k, 1)$. This completes the proof of the lemma.

The next lemma directly follows from the mean curvature comparison principle.

Lemma 4.5 Suppose $\Sigma \subset A$ is a compact domain such that: $f(\partial \Sigma)$ is contained in $\mathbb{B}(z, \eta)$, where $z \in \mathbb{S}^{2}$ and $\eta<\frac{1}{H_{0}}$. Then either $f(\Sigma) \subset \mathbb{B}(z, \eta)$ or $f(\Sigma)$ contains a point outside of $\mathbb{B}\left(z, \frac{1}{H_{0}}\right)$.

\section{Proof of the Theorem 1.1 .}

By Lemma 3.4, $L(A) \subset \mathbb{S}^{2}$ contains at least two distinct points $x$ and $y$. We next prove that the limit set of $f$ is the entire sphere $\mathbb{S}^{2}$. 
Lemma 5.1 $L(A)=\mathbb{S}^{2}$.

Proof. By Lemma 3.4, there are distinct points $x, y \in L(A) \subset \mathbb{S}^{2}$. Arguing by contradiction, suppose that there exists a point $p \in \mathbb{S}^{2}-L(A)$. The definition of limit point and the fact that $f: A \rightarrow \mathcal{D}$ is proper with $L(A) \subset \mathbb{S}^{2}$ imply there exists an $\varepsilon>0$ such that $\mathbb{B}(p, 10 \varepsilon) \cap f(A)=\varnothing$. By properness of $f$ in $\mathbb{B}$, then for $n$ large, we have $f\left(\overline{A-A_{n}}\right) \subset[\mathbb{B}-\overline{\mathbb{B}(1-\varepsilon)}]$.

Note that for some $\delta \in\left(0, \frac{1}{8} \varepsilon\right)$ sufficiently small, there exists a compact embedded annulus of revolution $E(\delta) \subset[(\overline{\mathbb{B}}-\mathbb{B}(1-\delta)) \cap \mathbb{B}(p, \varepsilon)]$ with boundary circles in $\mathbb{S}^{2} \cup\left[\mathbb{S}^{2}(1-\delta)\right]$ and such that the radial projection $r(E(\delta)) \subset \mathbb{S}^{2}$ is contained in the disk $\mathbb{B}(p, \varepsilon) \cap \mathbb{S}^{2}$. Furthermore, $E(\delta)$ is also chosen to have mean curvature greater than $H_{0}$ and with mean curvature vector outward pointing from the domain in $[\bar{B}-\mathbb{B}(1-\delta)]-E(\delta)$ which is contained in $\mathbb{B}(p, \varepsilon)$; for instance, $E(\delta)$ can be chosen to be a piece of a suitably scaled compact embedded annulus in some nodoid of constant mean curvature one, see Figure 3 Left. Assume now that $\varepsilon$ is also chosen less than $\frac{1}{10} d(x, y)$.

Assume that $n$ and $j$ are chosen sufficiently large so that:

1. $f(A(n, j)) \subset[\mathbb{B}-\overline{\mathbb{B}(1-\delta)}]$.

2. Any circle in $\mathbb{S}^{2}-\{x, y\}$ which represents the generator of the first homology group $\mathbb{H}_{1}\left(\mathbb{S}^{2}-\right.$ $\{x, y\})$ and whose distance from $x$ and $y$ is at least $\delta$, intersects the radial projection $r(f(A(n, j))) \subset$ $\mathbb{S}^{2}$. This property holds since $x$ and $y$ are limit points of $f(A)$.

3. The radial projection of each of the two boundary curves of $f(A(n, j))$ has diameter less than $\varepsilon$. This condition is possible to achieve since each of the components of $\partial f(A(n, j))$ has image on either a disk component of $\mathcal{W}$, a face of $\mathcal{F}$ or a component of $\partial \widetilde{N}(\Gamma)-\cup \mathcal{W}$, and each of these components and faces is contained ambient balls of radius $4 \delta$ by Lemma 2.2 , which in turn have radial projections of diameter less than $\varepsilon$.
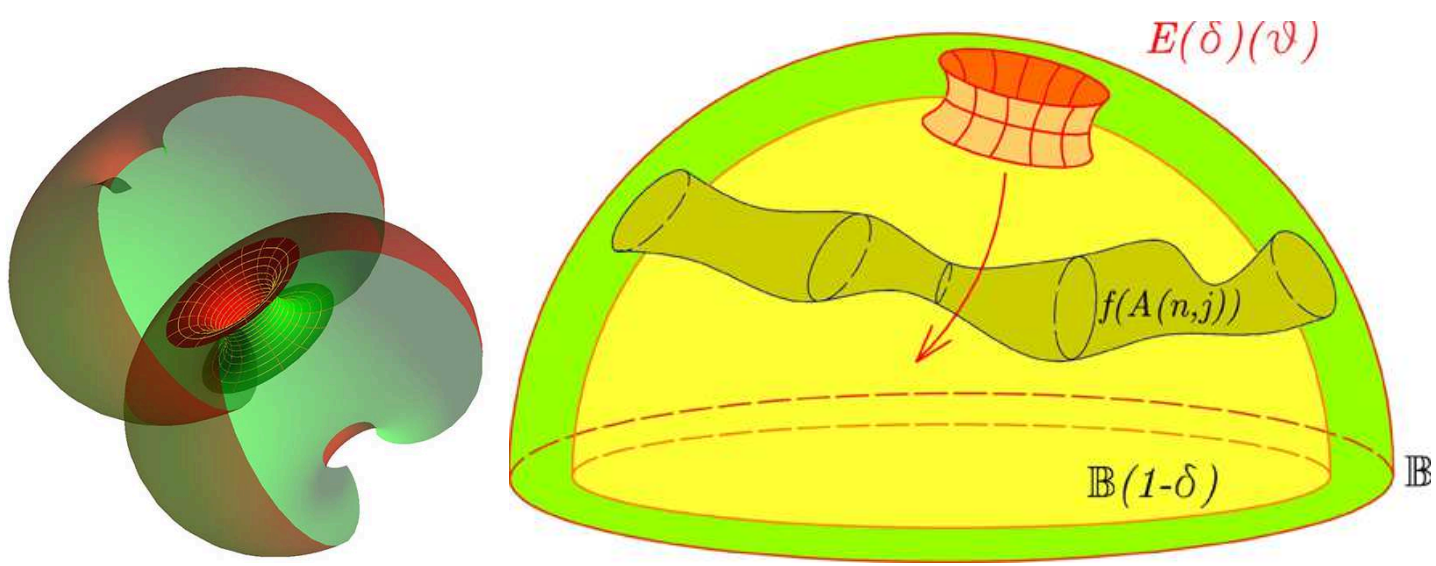

Figure 3: Left: This figure shows a domain on the nodoid corresponding to a scaling of $E(\delta)$. Right: Since all of the $E(\delta)(\vartheta)$ are disjoint from $\partial f(A(n, j))$, a first point of contact in $E(\delta)\left(\vartheta_{0}\right) \cap f(A(n, j))$ occurs an interior point of $f(A(n, j))$.

By the above three properties and our choices of $\varepsilon$ and $\delta$, we can choose a circle $S^{1} \subset \mathbb{S}^{2}-\{x, y\}$ which intersects $r(f(A(n, j)))$, and such that the $\varepsilon$-neighborhood $S^{1}(\varepsilon)$ of $S^{1}$ is disjoint from the 
radial projection $r(\partial f(A(n, j))) \subset \mathbb{S}^{2}$ and each component of $\mathbb{S}^{2}-r\left(S^{1}(\varepsilon)\right)$ contains points of $r(f(A(n, j)))$. Let $L$ be an oriented radial ray which is an axis for the circle $S^{1}$. For $\vartheta \in[0,2 \pi)$, consider the family of annuli $E(\delta)(\vartheta)$ obtained by rotating $E(\delta)$ counterclockwise around $L$ by the angle $\vartheta$. By elementary separation properties, there is a smallest $\vartheta_{0} \in(0,2 \pi)$ such that $E(\delta)\left(\vartheta_{0}\right) \cap$ $f(A(n, j)) \neq \varnothing$. Since all of the $E(\delta)(\vartheta)$ are disjoint from $\partial f(A(n, j))$, a first point of contact in $E(\delta)\left(\vartheta_{0}\right) \cap f(A(n, j))$ occurs an interior point of $f(A(n, j))$, which must have absolute mean curvature on $A$ at least equal to the minimum of the mean curvature of $E(\delta)\left(\vartheta_{0}\right)$ (see Figure 3 Right). But the mean curvature of $E(\delta)\left(\vartheta_{0}\right)$ is greater than the absolute mean curvature function of $A$. This contradiction completes the proof of Lemma 5.1

The next lemma follows immediately from the arguments presented in the proof of Lemma 5.1 also see Figure 3 Right. We note that the constant $H_{0}$ in the statement of the next lemma is the same constant which is the strict upper bound on the supremum of the absolute mean curvature of $f: A \rightarrow \mathbb{B}$.

Lemma 5.2 Given any $\varepsilon \in\left(0, \frac{1}{4}\right)$, there exists an $\eta_{0} \in\left(0, \frac{\varepsilon}{10}\right)$ that also depends on $H_{0}$ such that the following statements hold. For any $\eta \in\left(0, \eta_{0}\right]$ and for any immersion $g: \Sigma \rightarrow \mathbb{B}-\mathbb{B}(1-\eta)$ of a compact surface with boundary and absolute mean curvature less than $H_{0}$ such that $g(\partial \Sigma) \subset$ $[\overline{\mathbb{B}}(x, \eta) \cup \overline{\mathbb{B}}(y, \eta)]$ for two points $x, y \in \mathbb{S}^{2}$ with $d(x, y) \geq \varepsilon$, then either $g(\Sigma) \subset[\overline{\mathbb{B}}(x, \eta) \cup \overline{\mathbb{B}}(y, \eta)]$ or $g(\Sigma)$ is $\varepsilon$-close to every point in $\mathbb{S}^{2}$. (Note that it may be the case that $g(\partial \Sigma)$ is contained entirely in one of the balls $\overline{\mathbb{B}}(x, \eta), \overline{\mathbb{B}}(y, \eta)$.)

Lemma 5.3 Given an $\varepsilon \in\left(0, \frac{1}{2 H_{0}}\right)$, there exists an $n(\varepsilon) \in \mathbb{N}$ such that for each $k \geq n(\varepsilon)$, there exists a point $y(k) \in \mathbb{S}^{2}$ with $f(A(k, 1)) \subset \mathbb{B}(y(k), \varepsilon)$ and $f(A(n(k)) \subset[\mathbb{B}-\overline{\mathbb{B}}(1-\varepsilon)]$.

Proof. Fix $\varepsilon \in\left(0, \frac{1}{2 H_{0}}\right)$ and let $\eta=\min \left\{\eta_{0}, \eta_{1}\right\}$ where $\eta_{0}$ is given in Lemma 5.2 and depends on $\varepsilon$ and $H_{0}$ and $\eta_{1}$ given in Lemma 4.2 Let $k(\eta)$ be the related integer given in Lemma 4.4. We claim that for $k \geq k(\eta), f(A(k, 1)) \subset \mathbb{B}(z(k), \eta)$ and that $f(A(k(\eta)) \subset[\mathbb{B}-\overline{\mathbb{B}}(1-\varepsilon)]$, and so, by setting $n(\varepsilon)=k(\eta)$, this claim will complete the proof of the lemma. By Lemma 4.4, $f(A(k(\eta))) \subset$ $[\mathbb{B}-\overline{\mathbb{B}}(1-\eta)] \subset \mathbb{B}-\bar{B}(1-\varepsilon)$ and so it remains to verify that $f(A(k, 1)) \subset \mathbb{B}(z(k), \eta)$.

Suppose that $f(A(k, 1))$ contains a point outside of $\mathbb{B}(z(k), \eta)$. Let $K$ be a nonempty component in $\left(f_{A(1, k)}\right)^{-1}(\mathbb{B}-\mathbb{B}(z(k), \eta))$. By Lemma 4.5, there is a point on $K$ which lies outside of $\mathbb{B}\left(z(k), \frac{1}{H_{0}}\right)$. Since $\varepsilon \in\left(0, \frac{1}{2 H_{0}}\right)$ and $\eta \leq \eta_{0}$, Lemma 5.2 implies that the distance between every point of $\mathbb{S}^{2}$ and $K$ is at most $\varepsilon$. In particular, there exists a point $p \in K$ such that $f(p)$ has distance greater than 1 from $z(k)$.

By the third statement in Lemma 4.4 each boundary curve of $K$ bounds a disk in $A(k, 1)$. From the simple topology of an annulus we find that exactly one boundary curve of $K$ bounds a disk $D \subset$ $A(k, 1)$ and such that $K \subset D$. Next we apply Lemma 4.2 to find an $X_{i}$-type curve $\beta_{1} \subset D$ which bounds a subdisk $D\left(\beta_{1}\right)$ which contains the point $p$ and which satisfies the other properties in that lemma. In particular, we may assume the intrinsic distance from $D\left(\beta_{1}\right)$ to $\partial D$ is at least $\eta$. By the second statement in Lemma 4.4 $f\left(\beta_{1}\right) \subset \mathbb{B}\left(y\left(\beta_{1}\right), \eta\right)$ for some point $y\left(\beta_{1}\right) \in \mathbb{S}^{2}$. By our previous arguments there exists a point $p_{1} \in D\left(\beta_{1}\right)$ such that the distance from $f\left(p_{1}\right)$ to $y\left(\beta_{1}\right)$ is greater than one. So, we can apply Lemma 4.2 again to obtain a subdisk $D\left(\beta_{2}\right), D \supset D\left(\beta_{1}\right) \supset D\left(\beta_{2}\right)$, where the intrinsic distance from $\partial D\left(\beta_{1}\right)$ to $D\left(\beta_{2}\right)$ is at least $\eta$. Repeating these arguments, induction gives the existence of a sequence of disks $D \supset D\left(\beta_{1}\right) \supset \ldots \supset D\left(\beta_{n}\right) \supset \ldots$ such that the intrinsic distance from $D\left(\beta_{n}\right)$ to $\partial D$ is at least $n \eta$. Since $D$ is compact, we obtain a contradiction which proves our 
earlier claim that $f(A(k, 1)) \subset \mathbb{B}(z(k), \eta)$ for $k \geq k(\eta)$. As we have already observed, this claim then proves the lemma.

We now complete the proof of Theorem 1.1. Fix some $\varepsilon^{\prime} \in\left(0, \frac{1}{2 H_{0}}\right)$ and let $\eta_{0} \in\left(0, \frac{\varepsilon^{\prime}}{10}\right)$ be the related number given in Lemma 5.2 Set $\varepsilon=\eta_{0}$ and let $n(\varepsilon)$ be the integer given in Lemma 5.3. By Lemma 5.3, for each $i \in \mathbb{N}, f(A(n(\varepsilon), i)) \subset\left[\mathbb{B}-\mathbb{B}\left(1-\eta_{0}\right)\right], f\left(\gamma_{n(\varepsilon)}\right) \subset \mathbb{B}\left(y(n(\varepsilon)), \eta_{0}\right)$ and $f\left(\gamma_{n(\varepsilon)+i}\right) \subset \mathbb{B}\left(y(n(\varepsilon)+i), \eta_{0}\right)$. Since the limit set of $A(n(\varepsilon))$ is all of $\mathbb{S}^{2}$, there exists a smallest $j \in \mathbb{N}$ such that the distance between $y(n(\varepsilon))$ and $y(n(\varepsilon)+j)$ is greater that $\varepsilon^{\prime}$. By Lemma 5.2 and taking into account that $\mathbb{B}\left(y(n(\varepsilon)), \eta_{0}\right)$ and $\mathbb{B}\left(y(n(\varepsilon)+j), \eta_{0}\right)$ are disjoint, then we conclude that $f(A(n(\varepsilon), j))$ must be $\varepsilon^{\prime}$ close to every point of $\mathbb{S}^{2}$.

On the other hand, given $k \in \mathbb{N}, n(\varepsilon) \leq k<n(\varepsilon)+j$, we know (by Lemma5.3) that $f(A(k, 1)) \subset$ $\mathbb{B}(y(k), \varepsilon)$, for a suitable $y(k) \in \mathbb{S}^{2}$. Moreover, the choice of $j$ implies that $f\left(\gamma_{k}\right) \subset \mathbb{B}\left(y(n(\varepsilon)), \varepsilon^{\prime}+\right.$ $\varepsilon)$, for $k$ satisfying $n(\varepsilon) \leq k<n(\varepsilon)+j$. So, by the triangle inequality we deduce $f(A(k, 1)) \subset$ $\mathbb{B}\left(y(n(\varepsilon)), \varepsilon^{\prime}+2 \varepsilon\right) \subset \mathbb{B}\left(y(n(\varepsilon)), 2 \varepsilon^{\prime}\right)$ for any $k$ satisfying $n(\varepsilon) \leq k<n(\varepsilon)+j$. This implies $f(A(n(\varepsilon), j)) \subset \mathbb{B}\left(y(n(\varepsilon)), 2 \varepsilon^{\prime}\right)$ which is impossible since $2 \varepsilon^{\prime}<\frac{1}{10}$ and we have already seen that $f(A(n(\varepsilon), j))$ must be $\varepsilon^{\prime}$ close to every point of $\mathbb{S}^{2}$. This contradiction completes the proof of Theorem 1.1 in the case $\bar{W}=\overline{\mathbb{B}}$.

For the general case where $\bar{W}$ is a smooth compact Riemannian manifold with nonempty boundary, small modifications of the proof of Theorem 1.1 in the special case $\bar{W}=\overline{\mathbb{B}} \subset \mathbb{R}^{3}$ also demonstrate that there exists a properly embedded 1-manifold $\Delta_{W} \subset W$, whose path components are smooth simple closed curves, such that $\mathcal{D}=W-\Delta_{W}$ is a Calabi-Yau domain for any open surface with at least one annular end. In carrying out these modifications in the smooth compact 3-manifold $\bar{W}$, it is convenient, to place the 1-manifold $\Delta_{\bar{W}}$ in the union of small pairwise disjoint closed $\varepsilon$-neighborhoods of the boundary components of $\bar{W}$ which have a natural product structure derived from the distance function to the boundary component. The product structure simplifies the construction of the related 1-complex $\Gamma_{\bar{W}}$ which has one component in each of the $\varepsilon$-neighborhoods of each boundary component of $\bar{W}$. Also note that the properness of any proper immersion of $A=\mathbb{S}^{1} \times[0, \infty)$ into $\mathcal{D}$ guarantees that $A$ has a end representative which maps into the $\varepsilon$-neighborhood of exactly one of the boundary components of $\bar{W}$. This discussion completes the proof of Theorem 1.1

Remark 5.4 The reader familiar with the paper [6] might consider the question: Are the domains $\mathcal{D}_{\mathcal{F}} \subset \mathbb{R}^{3}[6]$, obtained by removing a infinite proper family $\mathcal{F}$ of horizontal circles from $\mathbb{B}$, CalabiYau domains for surfaces with at least one annular end? The answer to this question is no because for at least one such $\mathcal{D}_{\mathcal{F}}$ constructed in [6], there exists a proper, conformal, complete embedding $f: \mathbb{R}^{2} \rightarrow \mathcal{D}$ with absolute mean curvature function less than $1, f\left(\mathbb{R}^{2}\right)$ is a surface of revolution with axis the $x_{3}$-axis, $f\left(\mathbb{R}^{2}\right)$ has intrinsic linear area growth and has limit set $L\left(\mathbb{R}^{2}\right)=\mathbb{S}^{2}$. The mean curvature function of $f\left(\mathbb{R}^{2}\right)$ in this case contains points of mean curvature arbitrarily close to 1 and also arbitrarily close to -1 . In this case for $\mathcal{D}$, the circles in $\mathcal{F} \subset \mathbb{B}$ are chosen to have axis the $x_{3}$-axis; the surface has the appearance of taking an infinite connected sum of the spheres $\mathbb{S}_{k}^{2}, k \in \mathbb{N}$, defined at the beginning of Section 2, joined by small catenoidal type necks centered along points along the $x_{3}$-axis which limit to the north and south poles of $\mathbb{S}^{2}$.

We conclude the paper with the following conjecture.

Conjecture 5.5 Let $\Delta \subset \mathbb{B}$ be the properly embedded one-manifold given in the proof of Theorem 1.1 If $\bar{B}$ is a smooth compact Riemannian three-ball and $F: \bar{B} \rightarrow \mathbb{B}$ is a smooth diffeomorphism, then $\mathcal{D}=B-F^{-1}(\Delta)$ is a Calabi-Yau domain for any noncompact surface with compact boundary 
(possibly empty). In particular, $\mathcal{D}=\mathbb{B}-\Delta$ does not admit any complete, properly immersed open surfaces with bounded mean curvature.

Francisco Martín at fmartin@ugr.es

Department of Geometry and Topology, University of Granada, 18071 Granada, Spain

William H. Meeks, III at bill@ math.umass.edu

Mathematics Department, University of Massachusetts, Amherst, MA 01003 USA

\section{References}

[1] A. Alarcón, L. Ferrer and F. Martín. Density theorems for complete minimal surfaces in $\mathbb{R}^{3}$. G.A.F.A., 18:1-49, 2008, MR2399094, Zbl 1144.53013.

[2] E. Calabi. Problems in differential geometry. Ed. S. Kobayashi and J. Ells, Jr., Proceedings of the United States-Japan Seminar in Differential Geometry, Kyoto, Japan, 1965. Nippon Hyoronsha Co., Ltd., Tokyo (1966) 170.

[3] R. Harvey and B. Lawson, Extending minimal varieties, Invent. Math., 28:209-226, 1975, MR0370319, Zbl 0316.49032

[4] L. Ferrer, F. Martín and W. H. Meeks III. Existence of proper minimal surfaces of arbitrary topological type. Preprint, 2009, arXiv:0903.4194.

[5] W. H. Meeks III. Proofs of some classical results in minimal surface theory. Indiana J. of Math., 54(4):1031-1045, 2005, MR2164416, Zbl 1085.53010.

[6] F. Martín, W. H. Meeks III, and N. Nadirashvili. Bounded domains which are universal for minimal surfaces. American Journal of Math., 129(2):455-461, 2007, MR2306042, Zbl pre05152573.

[7] F. Martín and S. Morales. On the asymptotic behavior of a complete bounded minimal surface in $\mathbb{R}^{3}$. Trans. of the A.M.S., 356(10):3985-3994, 2004, MR2058515, Zbl 1057.53009.

[8] F. Martín and S. Morales. Complete proper minimal surfaces in convex bodies of $\mathbb{R}^{3}$. Duke Math. J., 128(3):559-593, 2005, MR2145744, Zbl 1082.53009.

[9] F. Martín and S. Morales. Complete proper minimal surfaces in convex bodies of $\mathbb{R}^{3}$ (II): The behaviour of the limit set. Comment. Math. Helv., 81:699-725, 2006, MR2250860, Zbl 1110.53007 .

[10] N. Nadirashvili. Hadamard's and Calabi-Yau's conjectures on negatively curved and minimal surfaces. Invent. Math., 126(3):457-465, 1996, MR1419004, Zbl 0881.53053.

[11] S.-T. Yau, Problem section. Seminar on Differential Geometry, pp. 669-706, Ann. of Math. Stud., 102, Princeton Univ. Press, Princeton, N.J., 1982, MR0645762, Zbl 0479.53001.

[12] S.-T. Yau, Review of geometry and analysis. Mathematics: frontiers and perspectives, 353-401, Amer. Math. Soc., Providence, RI, 2000, MR1754787, Zbl 0969.53001. 